\title{
O desenvolvimento da teoria quântica no contexto da cultura de Weimar e o debate em torno das "teses de Forman"
}

\author{
Francisco Assis de Queiroz \\ Professor Departamento de História \\ USP \\ frantota@uol.com.br
}

\author{
Francisco Rômulo Monte Ferreira \\ Professor Instituto de Bioquímica Médica \\ UFRJ \\ fromulo@usp.br
}

Como citar este artigo: Queiroz, Francisco Assis, Rômulo M. F, Francisco "O desenvolvimento da teoria quântica no contexto da cultura de Weimar e o debate em torno das 'teses de Forman"'. Khronos, Revista de História da Ciência, no 8, pp. 50-63. 2019. Disponível em < http://revistas.usp.br/khronos>. Acesso em dd/mm/aaaa.

Resumo: A análise do desenvolvimento da teoria quântica na Alemanha a partir do final da Primeira Guerra Mundial é a tarefa empreendida por Paul Forman em A Cultura de Weimar, a Causalidade e a Teoria Quântica, 1918-1927. A partir da apresentação das ideias básicas de Forman, procura-se apreciar e analisar no presente ensaio sua repercussão e desdobramentos críticos em torno de suas teses.

Palavras-chave: Mecânica quântica, cultura de Weimar, causalidade, história da ciência.

The development of quantum theory in the context of culture Weimar and the debate around "Forman's theses"

\begin{abstract}
The analysis of the development of quantum theory in Germany from the end of the First World War is the task undertaken by Paul Forman in The Weimar Culture, Causality and Quantum Theory, 1918-1927. From the presentation of the basic ideas of Forman, seeks to appreciate and analyze in this test its impact and critical developments around their theses.
\end{abstract}

Keywords: quantum mechanics; Weimar culture; causality; history of science. 
A noção de lei científica se impõe no século XVII, com Descartes, Boyle, Newton, entre outros, quando a física se torna uma ciência autônoma em relação à filosofia, prosseguindo com a química no século XVIII, a biologia, a psicologia, geologia, geografia, economia, história, sociologia, linguística etc., no século XIX (período em que ocorre de forma aguda o processo de institucionalização e profissionalização das ciências). As ciências sociais, buscando constituírem-se como disciplinas científicas, positivas, pretendiam neutralizar a subjetividade do observador e eliminar qualquer juízo de valor da sua atividade. Assim, para Auguste Comte (1798-1857), o estudo da sociedade seria feito à semelhança do estudo da natureza, através da física social. E se as sociedades são regidas por leis, assim também é a história.

A mecânica clássica foi frequentemente associada a uma concepção determinista, muito ligada à noção de causalidade, de maneira necessária e rígida. Entretanto, as novas experiências e fenômenos estudados no final do século XIX até o surgimento da teoria da relatividade e, principalmente, da mecânica quântica, na primeira metade do século XX, provocaram uma transformação radical também em termos conceituais¹. Daí as noções correntes, hoje, de previsão provável ou probabilidade e indeterminismo.

O caráter relativo de tempo e espaço, bem como a indeterminação e as questões referentes à interferência do sujeito no ato de medição na mecânica quântica mudaram consideravelmente a perspectiva da ciência nas primeiras décadas do século XX.

Nesse período a física descobriu, na complexidade dos fenômenos elementares, a presença da acausalidade na natureza, como veremos mais à frente. Contudo, ela não é mais ou menos científica quando trabalha com probabilidades e explicações não determinísticas. Ademais, conforme Ilya Prigogine (1917-2003), ganhador do prêmio No-

\footnotetext{
${ }^{1} \mathrm{O}$ conhecimento maior do chamado estado sólido, da estrutura íntima da matéria tem apresentado problemas cruciais do ponto de vista filosófico, do conhecimento, da realidade, enfim, dando ensejo a acalorados debates, entre os quais está o famoso debate Einstein-Bohr entre 1925-1935 sobre a relação entre a teoria quântica e a realidade. Ela se constitui, assim, numa contribuição contemporânea fundamental para a ciência, a história e a cultura em geral. Ilustrativo a esse respeito e do diálogo possível entre as várias áreas de conhecimento é o livro O Direito Quântico: Ensaio sobre o Fundamento da Ordem Jurídica, do renomado jurista Goffredo Telles Júnior, publicado em 1971. Duas resenhas da obra foram publicadas na revista Ciência e Cultura, da Sociedade Brasileira para o Progresso da Ciência (SBPC). A primeira em janeiro de 1972, escrita pelo consagrado biólogo e jornalista José Reis e a segunda, em 1974, escrita por Henrique Fleming, então diretor do Instituto de Física da USP. Telles Jr. fala da evolução da vida e, em grande parte do livro, expõe as ideias principais da física moderna e contemporânea estudando, nas palavras de Fleming, as consequências da revolução conceitual introduzida pela Física Quântica sobre os Fundamentos do Direito.
} 
bel de Química de 1977, e Isabelle Stengers (1949- ), sistemas físicos abertos são produtos de sua história, na medida em que apresentam um caráter de irreversibilidade, de evolução².

O século XIX foi pródigo em termos de desenvolvimento das ciências, tendo sido até considerado por Comte como o momento em que a história e o conhecimento atingiram o estágio positivo (científico), abandonando suas concepções míticas e metafísicas. Desde então o cientificismo - a certeza e o otimismo com relação à ciência assumiu o caráter de uma crença bastante forte. Na análise do historiador Eric Hobsbawm (1917-2012), “A sociedade burguesa de nosso período (1848-1875) estava confiante e orgulhosa de seus sucessos. Em nenhum outro campo da vida humana isso era mais evidente que no avanço do conhecimento, da 'ciência"'3. Daí que muitos pesquisadores, entre os quais o físico Lord Kelvin, estavam convencidos de que, com exceção de dois problemas, o mundo físico era explicado basicamente pelas teorias de Newton e Maxwell e que "faltavam apenas detalhes a serem ajustados nessa ciência"4. Tais problemas eram precisamente o da radiação do corpo negro e o da negação do éter, os quais conduziram à teoria quântica e à teoria da relatividade, respectivamente ${ }^{5}$. A revolução que esses "detalhes” provocaram justifica o título expressivo, a esse respeito, de um dos capítulos do segundo livro citado de Hobsbawm: "Certezas solapadas: as ciências".

Em 1900 o físico alemão Max Planck (1858-1947), partindo do problema da radiação emitida por um corpo aquecido, propôs que a energia ou a radiação dos átomos não podia ser absorvida ou emitida continuamente, mas em pacotes discretos (descontínuos) ou quanta de energia. Isso significava que a energia, assim como a matéria, também era atômica. Essa teoria seria depois desenvolvida por muitos outros cientistas, com implicações profundas para a ciência e a filosofia contemporânea.

Esse foi o ponto de partida também para outra teoria revolucionária do começo do século XX, a teoria da relatividade - a relatividade especial ou restrita (1905) e a relatividade geral (1915) -, associada principalmente ao nome de Albert Einstein (1879-

\footnotetext{
2 PRIGOGINE, Ilya e STENGERS, Isabelle. A Nova Aliança: Metamorfose da Ciência. Tradução de Miguel Faria e Maria Joaquina Machado Trincheira; Brasília: Editora da UnB, 1991.

${ }^{3}$ HOBSBAWM, Eric J. A Era do Capital: 1848-1875. Tradução de Luciano Costa Neto; Rio de Janeiro: Paz e Terra, $5^{a}$ ed. rev., 2000, p. 349.

${ }^{4}$ HOBSBAWM, Eric J. A Era dos Impérios: 1875-1914. Tradução de Sieni Maria Campos e Yolanda Steidel de Toledo; Rio de Janeiro: Paz e Terra, 5a ed., 1998, p. 348.

${ }^{5}$ Aplicando também à luz a hipótese de Planck sobre a radiação do corpo negro, Einstein explicou, em 1905 (ano da formulação da relatividade restrita), o efeito fotoelétrico, que havia sido descoberto experimentalmente por Hertz em 1887.
} 
1955). Embora seja mais conhecido por essa teoria, Einstein trabalhou também em outros campos, como a teoria cinética da matéria e a teoria quântica da luz. Em conexão com essa teoria, em 1905, trabalhando em Berna, na Suíça, Einstein considerou que a energia luminosa também é distribuída descontinuamente no espaço, derivando daí a explicação do efeito fotoelétrico (fotoemissivo), trabalho que o fez receber o prêmio Nobel em 1921. Na verdade, Einstein mostrou como a teoria quântica de Planck poderia ser usada para explicar o efeito fotoelétrico, descrevendo a luz em termos de partículas (pacotes de energia) chamadas fótons. Cada uma dessas partículas tem uma energia $E$ dada pela relação de Planck-Einstein para a energia de um quantum de luz: $E=h f$ (sendo $h$ a constante de ação de Planck, e $f$ a frequência da luz).

No mesmo ano do trabalho sobre o efeito fotoelétrico, o texto básico da teoria da relatividade restrita foi publicado também em 1905 nos Anais de Física, com o título "Sobre a eletrodinâmica dos corpos em movimento", onde Einstein rejeitou as ideias de espaço estacionário absoluto e da existência do éter. Além disso, concluiu que nada poderia viajar mais depressa do que a velocidade da luz (trata-se na verdade de um pressuposto coerente à sua formulação). A relatividade restrita diz respeito apenas a corpos em movimento relativo entre si e com velocidades uniformes, ou seja, não acelerados. Foi ainda num outro texto de 1905 que ele estabeleceu a famosa relação da equivalência entre massa e energia expressa pela famosa fórmula $E=m c^{2}$, onde $c$ é a velocidade da luz. Essa equação é a expressão teórica da enorme quantidade de energia contida dentro do átomo.

$\mathrm{Na}$ medida em que incorporou o movimento acelerado e sendo este decorrência da gravidade, a relatividade geral (1915) se tornou também uma teoria da gravidade, uma reformulação da teoria newtoniana da gravitação. Ela alterou muitas das noções clássicas - espaço e tempo absolutos, espaço plano, matéria etc. -, baseadas na percepção cotidiana, ao falar de tempo relativo, espaço curvo etc. São noções que, para a grande maioria das pessoas, têm ainda um caráter muito obscuro e esotérico, devendo levar algum tempo para que sejam assimiladas mais racional e claramente. De qualquer forma, a teoria da relatividade geral teve uma série de provas que a validaram, entre as quais a determinação precisa da órbita de Mercúrio.

A nova mecânica quântica se desenvolveu principalmente na década de 1920 com contribuições de vários físicos, como Niels Bohr (1885-1962), Max Born (18821970), Louis de Broglie (1892-1987), Wolfgang Pauli (1900-1958), Erwin Schrödinger (1887-1961), Werner Heisenberg (1901-1976), Paul M. Dirac (1902-1984) entre outros. Além de seu complexo e relativamente bem estabelecido formalismo matemático, essa 
teoria tem sido objeto de diversas interpretações, apresentando consequências importantes sobretudo em termos de concepção de conhecimento quando, por exemplo, atribui um papel ativo ao observador, ao contrário do que ocorre na mecânica clássica. Isso significa que um sistema quântico evolui de maneira diferente, conforme seja observado ou não, enquanto o sistema clássico evolui do mesmo modo independente do sujeito.

O famoso princípio de incerteza ou indeterminação proposto por Heisenberg em 1927, ou seja, de que não é possível se medir com precisão absoluta, simultaneamente, a posição e o momento de uma partícula - quanto mais se conhece uma variável, menos se conhece a outra -, traria profundas implicações filosóficas, pois os sistemas quânticos, ao serem regidos pelas leis da probabilidade, indeterminação e acausalidade, colocavam em questão a percepção do senso comum e a base da ciência clássica dos sistemas mecânicos regidos pela causalidade e pelo determinismo.

Vale lembrar ainda que a mecânica quântica também tem obtido muito sucesso e contribuído de maneira considerável para o conhecimento do "átomo" e, consequentemente, para o conhecimento íntimo da matéria do estado sólido, importante para o desenvolvimento de materiais semicondutores para a fabricação de componentes e circuitos microeletrônicos com larga utilização na química, medicina, comunicação, informática etc. Suas implicações não seriam menores nos campos da epistemologia e do conhecimento em geral, constituindo-se num marco fundamental do desenvolvimento da ciência e da cultura contemporânea.

O trabalho de Paul Forman, A Cultura de Weimar, a Causalidade e a Teoria Quântica, 1918-1927: A adaptação de físicos e matemáticos alemães a um ambiente intelectual hostil, é um marco importante na historiografia da ciência contemporânea por sua abordagem e pela problemática que suscitou ${ }^{6}$. E isso precisamente porque, fugindo a uma mais tradicional abordagem internalista do desenvolvimento da ciência, ele adota uma perspec-

${ }^{6}$ FORMAN, Paul. A Cultura de Weimar, a Causalidade e a Teoria Quântica, 1918-1927: A adaptação de físicos e matemáticos alemães a um ambiente intelectual hostil; trad. de Cláudio Weber Abramo; Cadernos de História e Filosofia da Ciência: Centro de Lógica, Epistemologia e História da Ciência, Suplemento 2/1983, Unicamp. Analisaremos este trabalho a partir do artigo de John Hendry, "Weimar Culture and Quantum Causality" (History of Science, XVIII, 1980) e de outros trabalhos críticos que sumariaremos a partir da introdução que José Manuel Sánchez Ron faz na tradução espanhola do trabalho de Forman (em 1984). 
tiva externalista, ressaltando mais os fatores extrínsecos à própria ciência, como os sociais e culturais como seu condicionante e até mesmo determinante de seu desenvolvimento.

Forman é um dos principais historiadores norte-americanos da física contemporânea que, desde o final da década de 1960, vem se dedicando quase exclusivamente à análise e reconstrução da física alemã das primeiras décadas do século XX e, em especial, do período da República de Weimar?.

A reconstrução política, econômica e institucional do ambiente em que se praticava a física na Alemanha depois da Primeira Guerra Mundial já está contida na sua tese de doutorado, de 1967, cujo título é The Environment and Practice of Atomic Physics in Weimar Germany: A Study in the History of Science, que serviu de fonte para seus trabalhos posteriores. Em 1971 foi que apareceu, no terceiro volume de Historical Studies in the Physical Sciences, seu trabalho "Weimar Culture, Causality na Quantum Theory, 19181927”, que se tornou um clássico da historiografia da física a exemplo, em certo sentido, de um outro influente clássico que é o artigo do russo Boris Hessen, intitulado "The Social and Economic Roots of Newton's 'Principia”', apresentado no Segundo Congresso Internacional de História da Ciência e da Tecnologia, realizado em Londres, em 1931. Dizemos em certo sentido porque o trabalho de Hessen, não obstante pioneiro e muito interessante, apresenta uma leitura um tanto positivista e mecanicista das motivações econômicas nos Principia de Newton, o que, deve-se ressalvar, precisa ser entendido no contexto do marxismo soviético das décadas de 1920-308.

Quanto ao contexto do assunto ora em foco, convém lembrar, ainda que de maneira bastante sintética, que a República de Weimar (1919-1933) foi proclamada em Berlim em 9 de novembro de 1918, embora seu nascimento oficial seja assinalado pela promulgação de sua Constituição, em 11 de agosto de 1919, na cidade alemã de Weimar. Surgida de um movimento que derrubou o governo imperial alemão, era uma República de caráter social-democrático, tendo como presidente Friedrich Ebert (1919-1925), sucedido por Paul von Hindenburg (1925-1934).

Foi um período de inestimável contribuição em diversos ramos da cultura - o caso que estamos discutindo é apenas um exemplo. Mas a República teve que enfrentar,

7 SÁNCHEZ RON, José Manuel, na Introdução à versão espanhola por ele realizada, da obra de Forman (Alianza Editorial: Madrid, 1984), p. 17.

8 Uma tradução portuguesa do artigo de Hessen encontra-se no livro Ciência e Técnica: Antologia de Textos Históricos; São Paulo: T. A. Queiroz, 1992, organizado por Ruy Gama. Uma interessante análise desse trabalho foi realizada por Olival Freire Jr., em "Sobre 'As Raízes Sociais e Econômicas dos 'Principia' de Newton”, Revista da Sociedade Brasileira de História da Ciência, n. 9, pp. 51-64, 1993. 
entre outras coisas, a pesada dívida de guerra, crise econômica, com violenta desvalorização do marco, elevados índices de desemprego e inflação, divisões no governo em questões fundamentais, tentativas de restauração da monarquia por extremistas da direita, além dos efeitos da grande crise de 1929, iniciada nos Estados Unidos. Dessa forma, a débil República de Weimar acabou por sucumbir diante do agravamento da situação econômica alemã, abrindo espaço para à recepção da propaganda nazista e ascensão de Hitler ao cargo de chanceler em 30 de janeiro de 1933 e à presidência em 1934, quando da morte de Hindenburg9.

Em que medida esse contexto afetou ou não o trabalho dos cientistas, particularmente, no nosso caso, dos físicos e matemáticos? Tal é a tarefa empreendida por Forman. Conforme Sánchez Ron, três são o que os historiadores da ciência costumam distinguir e denominar como as "teses de Forman" presentes no seu ensaio. A primeira assinala a existência de um ambiente intelectual, na Alemanha de Weimar, antirracionalista e antipositivista, existencialista e neorromântico, cujo veículo de difusão foi o livro A Decadência do Ocidente, do historiador e filósofo alemão Oswald Spengler (1880-1936)

No próprio título do trabalho de Forman já estão contidos os elementos da tese que busca defender, qual seja, a de que o desenvolvimento da nova mecânica quântica acausal na Alemanha, substituindo a velha teoria quântica de Planck (1900), vai se dar em função da adaptação dos cientistas alemães a um ambiente intelectual do pós-guerra, marcado por uma hostilidade à causalidade e à racionalidade científico-tecnológica. ${ }^{10}$

Esse clima intelectual está referido no livro de Max Jammer, The Conceptual Development of Quantum Mechanics, que o autor cita no início, em que Jammer destaca as ideias filosóficas e correntes de pensamento do século XIX, como o contingentismo, o existencialismo e outras que teriam preparado o ambiente para o desenvolvimento da mecânica quântica moderna.

A pergunta de Forman é sobre o "por que e do como tais 'correntes de pensamento', cujo efeito sobre os físicos era evidentemente negligenciável na passagem do século, vieram a exercer uma influência tão forte sobre físicos alemães depois de

\footnotetext{
${ }^{9}$ Uma caracterização mais ampla desse contexto pode ser obtida a partir das seguintes obras: GAY, Peter, A Cultura de Weimar, trad. de Laura Lúcia da Costa Braga, Rio de Janeiro, Paz e Terra, 1978; RICHARD, Lionel, A República de Weimar, 1919-1933, São Paulo, Companhia das Letras: Círculo do Livro, 1988; THALMANN, Rita, A República de Weimar, trad. de Álvaro Cabral, Rio de Janeiro, Jorge Zahar Editor, 1988.

${ }^{10}$ SPENGLER, Oswald. A Decadência do Ocidente; Brasília: Editora da Universidade de Brasília.
} 
1918 "11. Se tais ideias não surgiram do nada, mas são frutos e se tornam compreensíveis a partir da realidade em que germinam e se desenvolvem, é desta realidade histórica, social e cultural que se deve partir.

Assim, o autor busca o trabalho de "exploração e reconstrução do ambiente intelectual de Weimar" nos historiadores da vida intelectual e comentadores contemporâneos, como Georg Lukács (1885-1971), Fritz Ringer (The Decline of the German Mandarins), Peter Gay (A Cultura de Weimar), entre outros, embora esses autores não tenham feito maiores considerações sobre a ciência. O próprio Peter Gay reconhece que "nada disse sobre a ciência... embora um dia planeje fazê-lo"12.

Partindo, então, da caracterização do ambiente cultural de Weimar como hostil à racionalidade científica em geral e, particularmente, ao determinismo e à causalidade - trata-se aqui da segunda tese -, Forman mostra como os físicos buscaram se acomodar e se adaptar ao estado de espírito do meio, abandonando suas concepções positivistas sobre a natureza da ciência, reformulando suas concepções e buscando recuperar seu prestígio social e intelectual.

Em função disso procura demonstrar, finalmente - terceira tese - a existência de um movimento relativamente bem difundido para eliminar a causalidade na física. Este movimento não se deve antes a desenvolvimentos internos da própria teoria quântica, mas decorre das influências externas, do meio, com relação à ciência. Deve-se destacar, ainda, que Forman utilizou como fontes para investigar as reações e perspectivas dos cientistas, aulas inaugurais, comunicados, conferências, tendo encontrado referências explícitas ou familiaridade com a obra de Spengler em Max Born (1882-1970), Albert Einstein, Franz Exner (1849-1926), Pascual Jordan (1902-1980), Richard von Mises (1883-1953) entre outros.

Em A Decadência do Ocidente (1918) Spengler elabora toda uma filosofia da história com extensas discussões sobre a ciência e um capítulo todo sobre a física intitulado "A Física faustiana e a Física apolínea", onde se refere à física como "a expressão e realização de certa cultura" ${ }^{3}$. Da mesma forma, o princípio da causalidade, como é tratado na matemática e na física, "é um fenômeno ocidental e, mais exatamente, barroco" ${ }^{14}$. Estão postas aí as teses de Spengler a respeito da não objetividade das ciências

\footnotetext{
11 FORMAN, op. cit., p. 6.

12 GAY, Peter. A Cultura de Weimar, trad. de Laura Lúcia da Costa Braga; Rio de Janeiro: Paz e Terra, 1978, p. 12.

13 SPENGLER, op. Cit., p.223.

14 SPENGLER, op. Cit., p.233.
} 
exatas. Como diz Forman, “(...) sua extensão de um relativismo cultural extremo à física e à matemática pretendia ser, e assim foi recebida, um desafio direto à ideologia dos cientistas exatos"15. Estes passam, sucessivamente, na expressão de Forman, a uma "capitulação ao spenglerismo", o que vai se refletir na sua própria produção, onde a palavra "crise" passa a ser constante, aparecendo explicitamente no título de vários artigos, como por exemplo, "A nova crise nos fundamentos da Matemática” de Hermann Weyl, "A atual crise na física alemã" de Johannes Stark (1922), "Sobre a crise do conceito de causalidade" de Joseph Petzoldt (1922) e até mesmo num artigo popular de Einstein "Sobre a atual crise na física teórica" (1922).

Forman mostra em seguida as sucessivas conversões à acausalidade por parte de cientistas, como Franz Exner (1919), Hermann Weyl (1920), Richard von Mises (1921), Walter Schottky (1921), Walther Nerst (1921), Hans Reichenbach (1925). Tentativas de fazer física acausal nessa época aparecem ainda em Hans Albrecht Senftleben e em um artigo publicado por Bohr, Kramers e Slater em 1924. Um caso particular aqui é o de Erwin Schrödinger que, “Tendo repudiado a causalidade em 1922-24 por razões sociais e éticas, por volta do outono de 1925 Schrödinger havia se reconvertido à causalidade por motivos que muito provavelmente eram pessoais e políticos”16.

De outro lado, a despeito de todas essas conversões, em 1922 e 1923, Planck e Einstein vão a público defender o princípio da causalidade, na física e fora dela. Wilhelm Wien saiu também em defesa da causalidade, procurando "afastar a tentativa de Spengler de descrever a física como culturalmente determinada" ${ }^{17}$. E no período de 1925-26 foi ele "quem novamente assumiu o papel de campeão da causalidade"18.

A conclusão de Forman, enfim, é que "problemas substanciais na física atômica desempenharam um papel apenas secundário na gênese do compromisso com a acausalidade; o fato mais importante foi a pressão sócio-intelectual exercida sobre os físicos, enquanto membros da comunidade acadêmica alemã"19. "Além de tudo, sejam quais forem as semelhanças que se possam encontrar na postura mental de cientistas exatos

\footnotetext{
${ }^{15}$ FORMAN, op. cit., p. 29-30.

${ }^{16}$ FORMAN, op. cit., p. 88.

${ }^{17}$ FORMAN, op. cit., p. 63.

${ }^{18}$ FORMAN, op. cit., p. 86.

${ }^{19}$ FORMAN, op. cit., p. 94.
} 
não-alemães nesse mesmo período, há uma peculiaridade que, penso, não é possível detectar fora da esfera cultural alemã: um repúdio à 'causalidade"'20.

\section{III}

John Hendry, em seu artigo intitulado "Weimar Culture and Quantum Causality", analisa o trabalho de Forman lembrando, inicialmente, que a história das ideias raramente é linear e que se deveria distinguir uma sutileza que Forman não distinguiu, ou seja, de que os ataques que a matemática e a física sofreram do exterior, do meio, eram em todos os casos ataques relativos ao seu valor em vez de sobre seu conteído ${ }^{21}$. Não há razão para supor, contudo, ressalva Hendry, que os físicos não reagiram aos ataques sobre o valor de sua disciplina adaptando seu conteúdo ${ }^{22}$. Hendry prossegue nas suas críticas. Segundo ele, os físicos e matemáticos eram, em algum grau, isolados do, em vez de atacados por forças do meio. ${ }^{23}$ Para ele, embora reconhecendo a possibilidade de isolamento, Forman concentra-se puramente sobre a adaptação ou capitulação ao meio, conduzindo, inevitavelmente, à produção de uma visão unilateral24.

Além disso, Hendry restringe o papel do meio na rejeição à causalidade, pois muitas das rejeições estavam encadeadas com os desenvolvimentos internos da física, não sendo verdade que a maioria tenha ardentemente desejado ou ativamente buscado uma mecânica quântica acausal. ${ }^{25}$ Nesse sentido, o critério de causalidade era apenas um aspecto da ideologia científica predominante; era o mais emocional e não o mais bá$\operatorname{sico}^{26}$.

Outros aspectos não explicitados por Forman, segundo Hendry, são os aspectos religiosos envolvidos no isolamento de físicos judeus, diferente de seus colegas cristãos, as influências individuais que tiveram os físicos, como por exemplo, de Husserl

\footnotetext{
${ }^{20}$ FORMAN, op. cit., pp. 97-98.

${ }^{21}$ HENDRY, John. Weimar Culture and Quantum Causality; History of Science, XVIII, 1980, p.157.

${ }^{22}$ HENDRY, op. cit., p. 158.

${ }^{23}$ HENDRY, op. cit., p. 158, itálicos do autor.

${ }^{24}$ HENDRY, op. cit., p.158.

${ }^{25}$ HENDRY, op. cit., p. 168.

${ }^{26}$ HENDRY, op. cit., p. 169.
} 
sobre Weyl, do filósofo existencialista do século XIX Kierkegaard sobre Bohr, do teólogo existencialista de Weimar Karl Barth sobre Kramers²7.

Para Hendry, mesmo negligenciando influências internas, o conceito de causação social é mais complexo do que Forman faz-nos acreditar, sendo que o trabalho dele demonstrou os perigos de um tratamento puramente externo e a pobreza de algum simples reducionismo social. Não se trata apenas de saber quais influências são mais importantes, mas como elas se combinam e se permeiam.

O próprio Hendry reconhece, no início de seu artigo, a validade das argumentações de Forman que, se não são completas e não dão conta totalmente das condições socioculturais e científicas para o desenvolvimento da nova mecânica quântica, não puderam, contudo, ser refutadas.

Quanto à ressalva de Hendry sobre a crítica aos cientistas exatos, em que medida se pode separar as críticas ao valor ou ao conteúdo de suas ciências, mesmo partindo de não especialistas, considerando que muitas pessoas poderiam ter acesso aos seus pressupostos básicos? De outro lado, Forman se concentrou na questão da causalidade, que era um dos pilares principais da ciência clássica e da cultura ocidental e como naquele momento de crise seu questionamento foi fundamental para ulterior desenvolvimento da mecânica quântica, o que não significa, como apontou Hendry, que outros fatores não estejam implicados. Ele indicou alguns, mas não os explicou. De que maneira fatores religiosos afetaram a relação dos físicos com o meio? Em que grau as influências individuais que os físicos sofreram afetaram suas concepções de um modo geral e científicas, em particular, um pouco na linha do trabalho referido de Gerald Holton sobre Bohr em "As raízes da complementaridade”? Que desdobramentos internos da própria física teriam contribuído para a conversão à acausalidade de um grande número de físicos, enquanto outros se mantiveram fiéis ao princípio da causalidade?

$\mathrm{Na}$ verdade, Forman analisa os fatores econômicos, políticos e culturais no desenvolvimento da ciência sem esquecer, porém, da sua autonomia relativa e da importância dos fatores internos no seu processo de desenvolvimento. Assim, quando afirma que o movimento para eliminar a causalidade na física não se deve antes a desenvolvimentos internos da própria teoria quântica, mas decorre das influências externas, do

${ }^{27}$ HENDRY, op. cit., p. 170. Sobre a influência de Kierkegaard sobre Bohr, cf. HOLTON, Gerald. "As Raízes da Complementaridade". Revista Humanidades, Brasília (UnB), 2 (9), out-dez., 1984, pp. 49-71 (traduzido por Dinorah de Oliveira Mendes, do original "The Roots of Complementarity", Daedalus, vol. 99, 1970). 
meio, em relação à ciência - ponto sobre o qual incide uma das críticas de Hendry -, ele não diz que os fatores internos não sejam importantes, mas sim que não são suficientes.

De outro modo, como entender reações tão diversas com relação à recepção da mecânica quântica acausal na Alemanha e na Inglaterra? A resposta pode ser encontrada em "The Reception of an Acausal Quantum Mechanics in Germany and Britain", que é uma extensão de “Weimar Culture...”. Este já contém a conclusão para o caso alemão, ou seja, "a aceitação do indeterminismo quântico na Alemanha é resultado - segundo Forman - de uma manifestação da ânsia dos cientistas por moldar as ciências exatas ao espírito da época, ao Zeitgeist dominante"28. Com relação à Inglaterra, "o meio ambiente intelectual não exercia pressões sobre os físicos britânicos e a causalidade não foi um tema importante anteriormente a 1925, pelo que o conteúdo epistemológico da mecânica quântica foi simplesmente passado por alto, sendo aceitos de maneira acrítica seu formalismo e a interpretação comumente aceita" 29.

\section{IV}

Outros trabalhos críticos do ensaio de Forman - além do artigo de Hendry que analisamos - são discutidos por Sánchez Ron, quais sejam: Hans Radder (1983), "Kramers and the Forman Thesis", History of Science, 21, 165-182 e P. Kraft e P. Kroes (1984), “Adaptation of Scientific Knowledge to an Intellectual Environment. Paul Forman's Weimar Culture, Causality and Quantum Theory, 1918-1927: Analysis and Criticism", Centaurus, 76-99. Como ele observa na nota 23: "Um quarto trabalho, de Jon Dorling, apresentado à British Society for the History of Science em julho de 1976, nunca chegou a ser publicado" 30 .

Enquanto Radder é mais um "revisionista", preocupado em estender as teses de Forman a cientistas não alemães, Hendry e Kraft-Kroes rejeitam algumas das principais conclusões de Forman e, especialmente, sua terceira tese. Já vimos, portanto, que Hendry restringe o papel do meio na rejeição à causalidade, vendo a introdução da acau-

${ }^{28}$ SÁNCHEZ RON, op. cit., p. 23.

${ }^{29}$ SÁNCHEZ RON, op. cit., p. 23.

${ }^{30}$ SÁNCHEZ RON, op. cit., p. 29. 
salidade na física quântica fundamentalmente como uma consequência de desenvolvimentos internos da própria física. Logo, para ele, não é verdade que a maioria dos físicos tenha ardentemente desejado ou ativamente buscado uma mecânica quântica acausal.

A seguir Sánchez Ron enumera as críticas contidas no artigo de Kraft e Kroes: “(1) Forman dá um peso excessivo ao papel desempenhado por grupos, associações etc., universitárias sim, porém formadas principalmente por pessoas sem a formação técnica suficiente como para poder entender o conteúdo das novas teorias ou propostas físicas. Este comportamento contrasta com o fato de que Forman não leva em conta as Reden (comunicações) apresentadas a audiências de cientistas profissionais. De fato, Kraft e Kroes argumentam que quando se faz isto se obtêm conclusões que não se podem reconciliar com as de Forman; (2) por que, se existia esse 'prestígio negativo' do qual constantemente fala Forman, as ciências físicas tiveram um financiamento tão alto durante o período em questão?; e (3) Forman supõe aparentemente que quantas e acausalidade são a mesma coisa, porém isto só foi assim depois de 1927; mais ainda, teorias como a de Bohr-Kramers-Slater, que continham propostas de mecanismos acausais, não foram bem-vindas, como Forman supõe, pelos físicos alemães”31.

A nosso ver o primeiro argumento de Kraft e Kroes soa como um argumento de autoridade. Será que essas audiências universitárias, com certa cultura, ainda que “sem a formação técnica suficiente”, não teriam acesso aos pressupostos básicos da nova ciência? Além disso, por que muitos cientistas profissionais se sensibilizaram e assimilaram as críticas à racionalidade, à causalidade e ao determinismo próprios de sua postura até então?

Quanto à segunda crítica, não parece haver contradição entre o ambiente hostil à causalidade - e consequente "prestígio negativo" dos cientistas - e o desenvolvimento científico, pois na Alemanha, ao contrário da França e da Inglaterra, havia já uma estreita relação entre ciência e indústria e apoio governamental antes da Primeira Guerra Mundial. Isso significa que aquele "prestígio negativo" não decorre de razões governamentais ou da falta de apoio privado à pesquisa, mas precisamente daquele clima geral da época, possivelmente catalisado por certos segmentos universitários e cultos em geral e expresso, em seu conjunto, no livro de Spengler já citado. Da mesma forma, as muitas

${ }^{31}$ SÁNCHEZ RON, op. cit., p. 30, itálicos do autor. 
críticas que se fazem hoje a certos desenvolvimentos da ciência e tecnologia não trazem como implicação necessária a redução de investimentos para sua investigação.

A terceira crítica é procedente, mas nos parece que Forman está mais preocupado com as influências gerais que fatores externos exerceram sobre o desenvolvimento da nova teoria quântica, do que com a não aceitação de uma eventual teoria que contivesse "propostas de mecanismos acausais". Contudo, é importante verificar porque teorias como a de Bohr-Kramers-Slater não foram bem-vindas pelos físicos alemães.

Se os trabalhos críticos que apresentamos são pertinentes, o trabalho de Forman continua, igualmente, instigante. Ambos são complementares e fundamentais para a compreensão de que, se a ciência possui uma autonomia relativa no seu desenvolvimento, não se pode, contudo, abstraí-la do quadro geral da sociedade e da cultura, já que ela é também um dos elementos da mesma sociedade e cultura. De qualquer forma, essas contribuições confirmam a repercussão e a importância do trabalho de Forman e a necessidade do debate permanente, pois a ciência, como elemento da cultura e, portanto, com uma dimensão histórica, está sempre em (re)construção. 